\title{
OPEN, FINITE TO ONE MAPS OF CONTRACTIBLE POLYHEDRA ONTO A SPHERE
}

GLEN E. BREDON

1. Introduction. For some time I have been concerned with the conjecture that an open and finite to one map $\phi: X \rightarrow Y$ between polyhedra must induce an epimorphism in rational homology. This is easily seen to be true in degree one. It is also known to hold for ramified coverings or for orbit maps of actions of finite groups because of the existence of "transfer homomorphisms" in these cases. (See [1] for example.)

In this note we present a counterexample to this conjecture by constructing an open, finite to one (in fact, at most three to one) map of a contractible polyhedron $K^{2}$ onto $S^{2}$. (By suspending, one obtains such maps onto $S^{n}$ for each $n \geqq 2$.) The branch locus on $S^{2}$ in our example is a triad and thus the map is a trivial 3-1 covering elsewhere.

Part of my interest in the conjecture was due to the fact, pointed out to us some time ago by $M$. Hirsch, that an affirmative answer would lead to a solution of a long standing problem concerning geodesics on surfaces (as to whether on a closed convex surface there must be a point for which all geodesics pass through another point). Hirsch noted that a counterexample to this geodesic problem would give rise to a 2-complex $K^{2}$ embedded in the unit tangent bundle of $S^{2}$ (or, just as well, in the Hopf bundle $S^{3}$ ) in such a way that the projection onto $S^{2}$ is finite to one and open from $K^{2}$ to $S^{2}$. Since $H_{2}\left(K^{2} ; Q\right) \rightarrow H_{2}\left(S^{2} ; Q\right)$ would then factor through zero, this would be a counterexample to our conjecture.

Requiring $K^{2} \rightarrow S^{2}$ to be embeddable in the Hopf map would place a restriction on the types of singularities which can occur in the map $K^{2} \rightarrow S^{2}$. For example, the map could not contain singularities of the type $z \mapsto z^{n}$ (of $D^{2} \rightarrow D^{2}$ ). Thus it is of value to give a counterexample which embeds in the Hopf map, and, in fact, we shall show that our example does admit such an embedding.

Shortly after hearing that I had constructed this example, Prabir Roy found another example which is an open map of the "dunce cap" onto $S^{2}$. We give a slightly simpler such map of the dunce cap in $\$ 4$. In Roy's example the branch locus on $S^{2}$ is an equator together with the poles and otherwise the map is $2-1$ on one hemisphere and $3-1$ on the other. In our example, the branch locus is an arc and a point and

Received by the editors December 18, 1968. 
the map is a 3-1 covering elsewhere. Neither of these examples on the dunce cap is contained in the Hopf map since both have singularities of the type $z \mapsto z^{n}$.

In $\$ 5$ we indicate a third example which shows that our conjecture remains false even when a rather strong local condition is placed on the singularities.

2. The main example. Consider three triangles $T_{1}, T_{2}$, and $T_{3}$. Subdivide each edge once and make the identifications of vertices and edges which are indicated in the top row of Figure 1. (If one folds up the corners of the triangles to form (cut) tetrahedra, so that the old edges form a triad, then the identifications are as indicated in the bottom row of Figure 1. This will indicate the branching over $S^{2}$ in a more convenient form.) The polyhedron resulting from these identifications is our $K^{2}$.
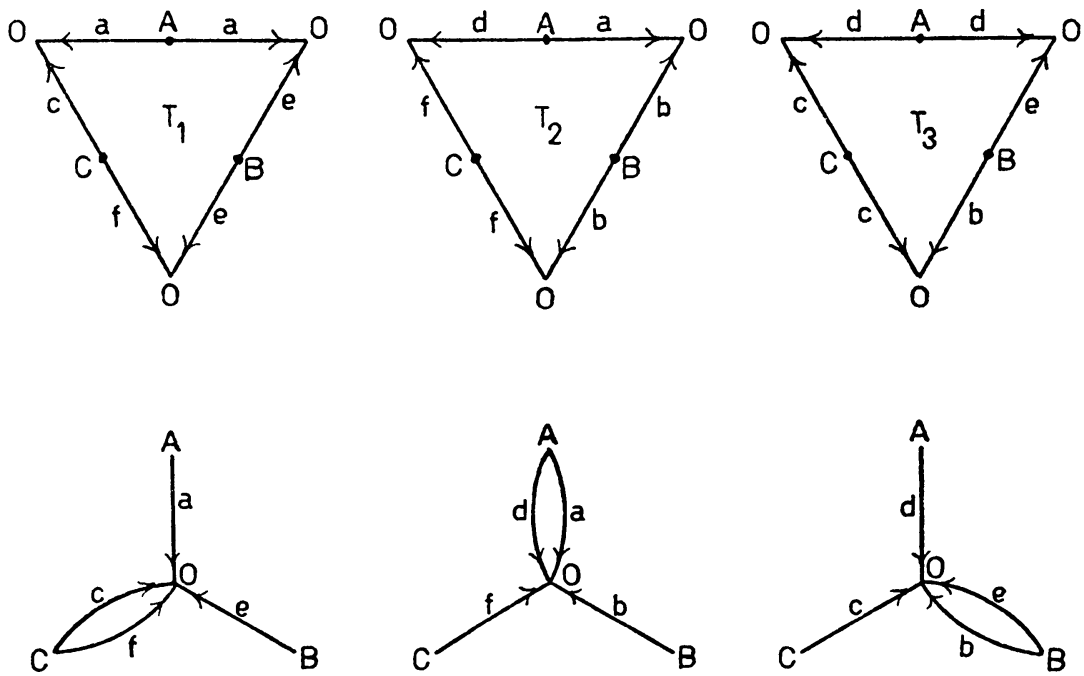

FIgURe 1

The map $\phi: K^{2} \rightarrow S^{2}$ is given by mapping each triangle $T_{i}$ onto a triangle $T$ and then folding up the corners of $T$ to form a tetrahedron (our $S^{2}$ ). This is indicated by Figure 2 where the vertices $O, A, B, C$ go to $O^{\prime}, A^{\prime}, B^{\prime}, C^{\prime}$ and the edges $a, d$ go to $a^{\prime} ; b, e$ go to $b^{\prime}$; and $c, f$ go to $c^{\prime}$. This map is clearly open and at most three to one. 

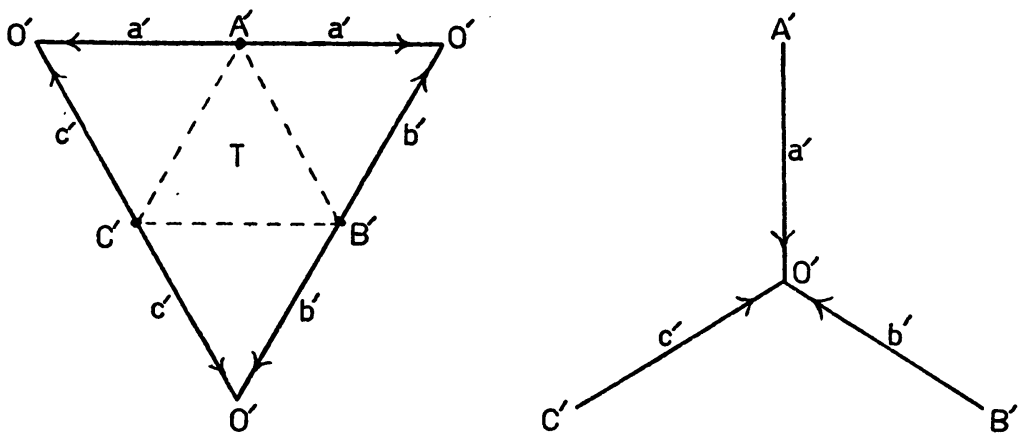

FIGURE 2

Now, with the vertex $O$ as a base point, $\pi_{1}(K)$ is clearly generated by $c^{-1} f, d^{-1} a$, and $b^{-1} e$. But in $T_{1}$ we see that $c^{-1} f=c^{-1} f e^{-1} e a^{-1} a=1$. Similarly $T_{2}$ and $T_{3}$ show that $d^{-1} a=1$ and $b^{-1} e=1$ so that $K$ is simply connected. A count of cells shows that $K$ has euler characteristic $3-6+4=1$ so that $H_{2}(K)$ must be trivial. Thus $K$ is contractible.

3. Embedding $\phi: K \rightarrow S^{2}$ in the Hopf map. Consider a disk $D^{2} C S^{2}$ about the point $O^{\prime}$ and containing the branch set. We shall first show how to embed $\phi^{-1}\left(D^{2}\right)$ in $D^{2} \times S^{1}$ so that $\phi$ is the projection of $D^{2} \times S^{1}$ on the first factor. We shall do this by looking closely at the portion of $K$ lying above circles about $O^{\prime}$. Note that $\phi^{-1}\left(O^{\prime}\right)$ is one point. Let $\lambda$ be a small circle in $S^{2}$ about $O^{\prime}$ and parametrize $\lambda$ in the clockwise direction (see Figure 3).

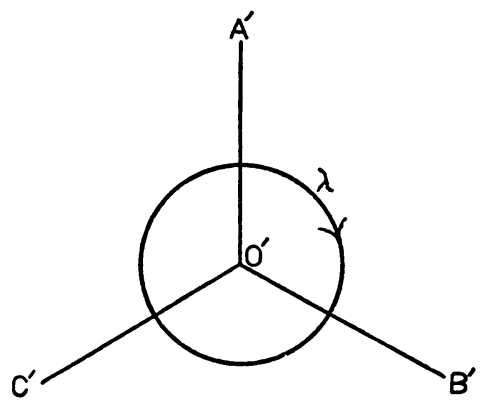

Figure 3

Then we see that $\phi^{-1}(\lambda)$ is as shown in Figure 4, where the left-hand side is identified with the right-hand side and $\phi$ is the vertical projection. The numbers indicate the branch $\left(T_{1}, T_{2}\right.$, or $\left.T_{3}\right)$ and the intersections with the edges $a, b, c, d, e, f$ are indicated. 


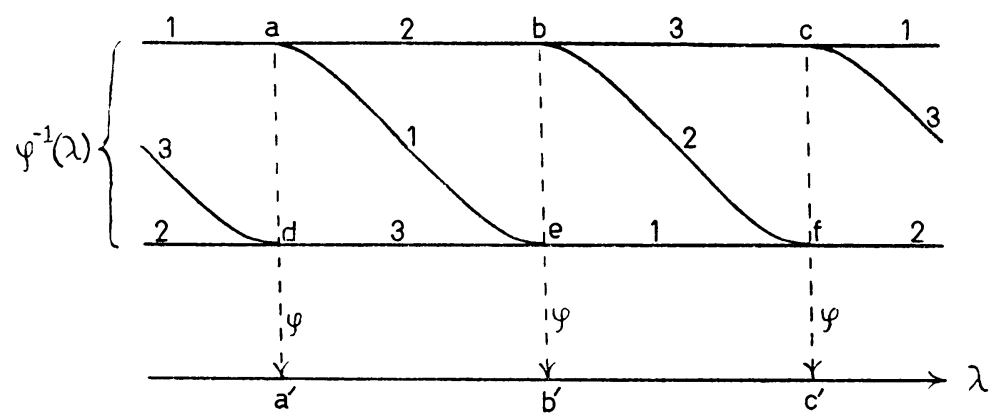

FIGURE 4

If $D_{\lambda}$ is the disk in $S^{2}$ about $O^{\prime}$ bounded by $\lambda$ then $\phi^{-1}\left(D_{\lambda}\right)$ is just the cone on $\phi^{-1}(\lambda)$. Since $\phi^{-1}(\lambda)$ embeds in $\lambda \times I$ this gives an obvious embedding of $\phi^{-1}\left(D_{\lambda}\right)$ in $D_{\lambda} \times I \subset D_{\lambda} \times S^{1}$.

Now we look at $\phi^{-1}(\lambda) \subset \lambda \times S^{1}$ from the slightly different viewpoint which is indicated in Figure 5 (i.e., rotate the $S^{1}$ coordinate).

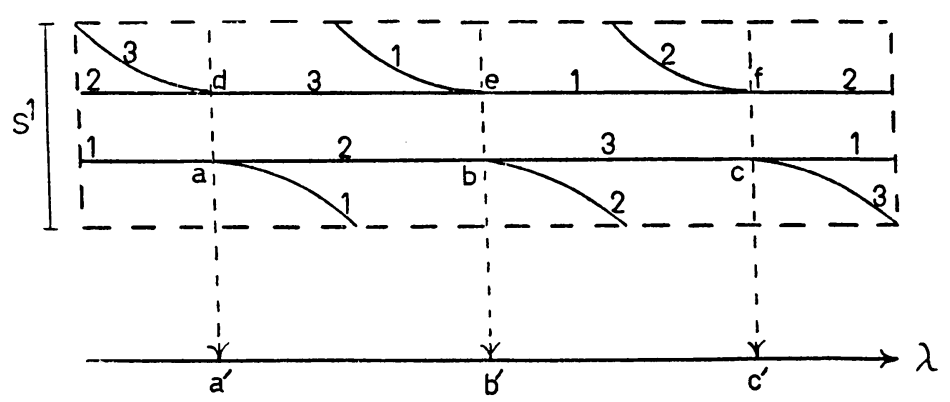

FIGURE 5

We let the radius of $\lambda$ increase so that $\lambda$ approaches the circle $\lambda_{1}$ about $O^{\prime}$ which passes through the vertices $A^{\prime}, B^{\prime}$ and $C^{\prime}$. While doing this we alter the embedding of $\phi^{-1}(\lambda)$ in $\lambda \times S^{1}$ indicated in Figure 5 by letting the points marked $a$ and $d$ approach each other, and similarly with $b, e$ and $c, f$. This produces an embedding $\phi^{-1}\left(D_{\lambda_{1}}\right) \subset D_{\lambda_{1}}$ $\times S^{1}$. The portion $\phi^{-1}\left(\lambda_{1}\right)$ is indicated in Figure 6. (The part lying in branch $T_{1}$ is dotted, that in branch $T_{2}$ is solid and that in branch $T_{3}$ is dashed.) 

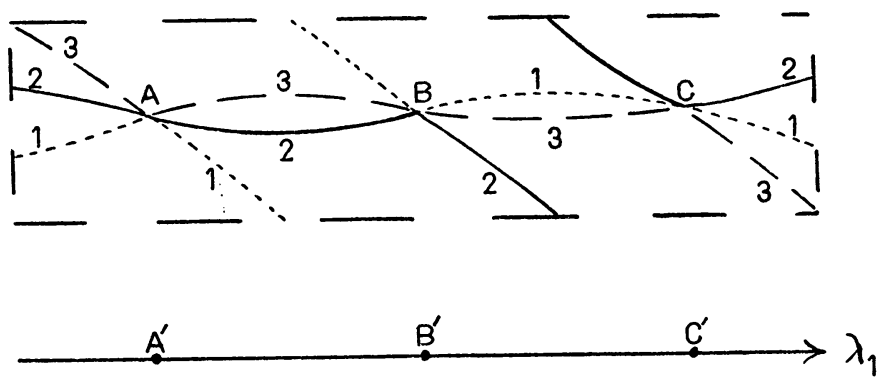

Figure 6

Now if we let $\lambda$ increase beyond $\lambda_{1}$ it is clear how to extend the embedding of $\phi^{-1}\left(D_{\lambda}\right)$ in $D_{\lambda} \times S^{1}$ so that for some $\lambda_{2}$ about $\lambda_{1}$ we have that $\phi^{-1}\left(\lambda_{2}\right)$ is as shown in Figure 7.

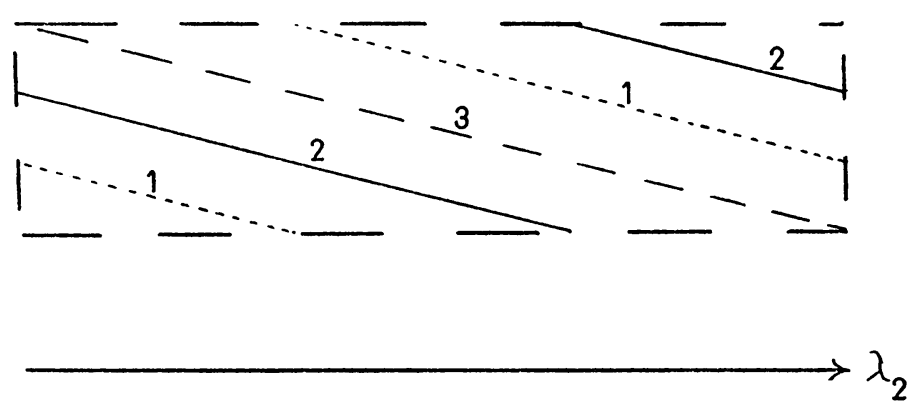

Figure 7

Let $D_{\lambda_{2}}^{\prime}$ be the complementary disk to $D_{\lambda_{2}}$ in $S^{2}$. We may attach $D_{\lambda_{2}} \times S^{1}$ to $D_{\lambda_{2}}^{\prime} \times S^{1}$ by a fiber preserving map on the boundaries (essentially $\left(z_{1}, z_{2}\right) \mapsto\left(z_{1}, z_{1} z_{2}\right)$ of $\left.S^{1} \times S^{1} \rightarrow S^{1} \times S^{1}\right)$ in such a way that the three curves in $\phi^{-1}\left(\lambda_{2}\right)$ are embedded horizontally with respect to the parametrization from $D_{\lambda_{2}}^{\prime} \times S^{1}$. These then extend to a trivial 3-fold covering of $D_{\lambda_{2}}^{\prime}$ in $D_{\lambda_{2}}^{\prime} \times S^{1}$. Since the $S^{1}$-bundle over $S^{2}$ arising from this attaching is just the Hopf bundle, this completes the required embedding of $\phi: K \rightarrow S^{2}$ in the Hopf map.

4. A map from the dunce cap. We now construct the open map of the dunce cap onto $S^{2}$ promised in the introduction. Recall that the dunce cap is the space obtained from the triangle by orienting the edges incoherently and then identifying all three. It is convenient for 
us to subdivide the edges first. Thus the dunce cap is the space indicated on the left of Figure 8. The map onto $S^{2}$ is given by merely iden-
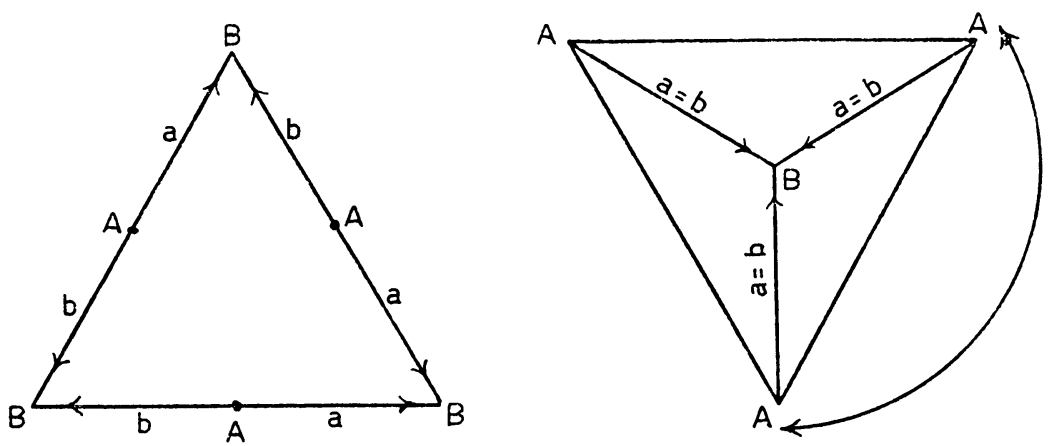

FIGURE 8

tifying all points in this triangle which are carried to one another by a rotation (120 degrees) of the triangle. (In particular, the edges $a$ and $b$ are identified.) The easiest way to see that the quotient space of this identification is $S^{2}$ is to note that it is obtained by folding up the corners of the triangle into a tetrahedron (see the right side of Figure 8 ) and then identifying under the indicated rotation of \pm 120 degrees. The branch set is clearly the arc forming the image of the edges $a$ and $b$ together with the point corresponding to the center of the triangle.

As was noted in the introduction, this example cannot be embedded in the Hopf map since the isolated branch point looks like $z \mapsto z^{3}$.

5. Another example. It would seem reasonable that if one makes strong restrictions on the type of singularities above points in $S^{2}$ for an open map $\phi: L^{2} \rightarrow S^{2}$ then $\phi_{*}: H_{2}\left(L^{2}\right) \rightarrow H_{2}\left(S^{2}\right)$ will be nontrivial.

The assumption that $\phi$ has a local cross section near any point of $S^{2}$ does not suffice as the example of $\$ 2$ shows. (In fact any finite to one, open, simplicial map contained in a circle fibration must admit local sections.) One might note, however, that in the example of $\$ 2$ the images of the cross sections defined on any given neighborhood of the point $O^{\prime}$ do not fill out a neighborhood of $\phi^{-1}\left(O^{\prime}\right)$ (and similarly with $A^{\prime}, B^{\prime}$, and $C^{\prime}$ ).

Suppose we call a point $p$ of $S^{2}$ "simple" for $\phi$ if there is a neighborhood $U$ of $p$ such that the images of the cross sections over $U$ fill out $\phi^{-1}(U)$. In this section we indicate an example of an open, finite to one, simplicial map $\phi: L^{2} \rightarrow S^{2}$ such that each point of $S^{2}$ is simple for 
$\phi$, but $\phi_{*}: H_{2}\left(L^{2}\right) \rightarrow H_{2}\left(S^{2}\right)$ is trivial. It seems to be difficult to come by such examples and the one we give is not contractible.

First, we define another contractible polyhedron $M^{2}$ and an open map $\psi: M^{2} \rightarrow S^{2}$. This is constructed in a similar manner to the construction of $K^{2}$ in $\$ 2$ with the only difference being that the branching over $S^{2}$ is to be as shown in Figure 9. Again it is easy to see that $M^{2}$ is
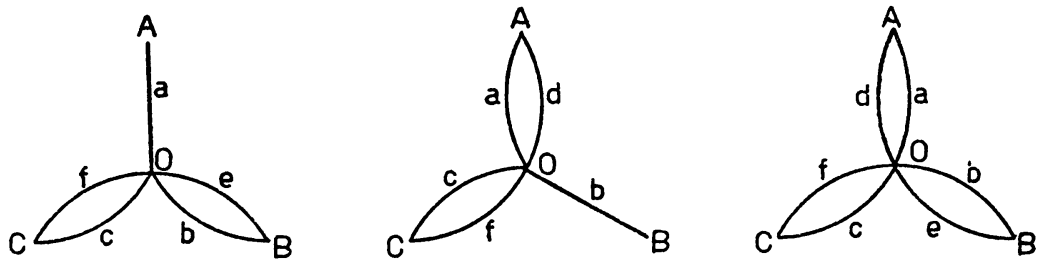

Figure 9

contractible and that the map $\psi$ onto $S^{2}$ is open. Moreover, the reader may verify that the point $O^{\prime}=\psi(0)$ in $S^{2}$ is simple for $\psi$. The points $A^{\prime}$, $B^{\prime}$, and $C^{\prime}$ are not simple. However, it is easily verified that there is a neighborhood $U \approx D^{2}$ of $A^{\prime}$ in $S^{2}$ (similarly $B^{\prime}$ and $C^{\prime}$ ) and a map $g: \psi^{-1}(U) \rightarrow D^{2}$ such that under the homeomorphism $U \approx D^{2}, x \mapsto \psi(x)$ becomes $x \mapsto g(x)^{2}$, and such that the global cross sections of $g$ fill out $\psi^{-1}(U)$.

Now consider the subgroup $Z_{2}+Z_{2} \approx G \subset S O$ (3) consisting of 180 degree rotations about the coordinate axes. The orbit space $S^{2} / G$ is homeomorphic to $S^{2}$. Thus the orbit map $S^{2} \rightarrow S^{2} / G$ may be regarded as an open map $h: S^{2} \rightarrow S^{2}$. Moreover, the branch locus of $h$ consists of three points which we may take to be $A^{\prime}, B^{\prime}$ and $C^{\prime}$. Each of these points has two inverse images under $h$ and on either branch $h$ looks like the map $x \mapsto x^{2}$ of $D^{2} \rightarrow D^{2}$.

Now we let $\phi: L^{2} \rightarrow S^{2}$ be the pull back of $\psi: M^{2} \rightarrow S^{2}$ under $h$ :

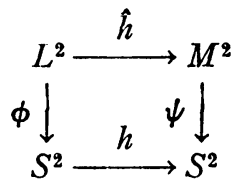

The reader may verify that every point of $S^{2}$ is simple for $\phi$ as claimed. Moreover, $4 \phi_{*}=h_{*} \phi_{*}=\psi_{*} \hat{h}_{*}=0$ so that $\phi_{*}=0$.

\section{REFERENCE}

1. G. E. Bredon, Sheaf theory, McGraw-Hill, New York, 1967. MR $36 \# 4552$.

RUTGERS UNIVERSITY 